\title{
Spontaneous Healing of Corneal Perforation after Temporary Discontinuation of Erlotinib Treatment
}

\author{
Naoyuki Morishige Nanami Hatabe Yukiko Morita Naoyuki Yamada \\ Kazuhiro Kimura Koh-Hei Sonoda \\ Department of Ophthalmology, Yamaguchi University Graduate School of Medicine, Ube, \\ Japan
}

\section{Key Words}

Erlotinib $\cdot$ Corneal perforation $\cdot$ Punctal plug

\begin{abstract}
Purpose: To report a case of corneal perforation associated with oral administration of erlotinib and its spontaneous healing after temporary discontinuation of drug treatment. Case Report: A 65-year-old man with metastatic lung cancer was treated with erlotinib (150 $\mathrm{mg} /$ day), a specific tyrosine kinase inhibitor of the epidermal growth factor receptor. He was referred to our corneal service for the treatment of bilateral corneal disorders, diagnosed with mild aqueous-deficient dry eye, and treated by insertion of punctal plugs. His corneal epithelial disorders initially improved, but subsequently worsened, as manifested by the development of bilateral corneal ulceration with corneal perforation in the right eye. The oral administration of erlotinib was interrupted in preparation for tectonic keratoplasty, but 2 days later the corneal perforation of the right eye and the bilateral epithelial defects had healed spontaneously. Treatment with erlotinib was resumed at half the initial dose, and the cornea of both eyes has remained apparently healthy. Discussion: Erlotinib may be secreted into tear fluid and thereby adversely affect the corneal epithelium. The development of corneal epithelial disorders in patients receiving this drug may be reversed by reducing its dose.


Morishige et al.: Spontaneous Healing of Corneal Perforation after Temporary Discontinuation of Erlotinib Treatment

\section{Case Report}

A 65-year-old Japanese man was diagnosed in April 2010 with stage IV lung adenocarcinoma, manifesting multiple metastases in the lung, adrenal gland and brain. He received 31 Gy of basicranial radiation for the brain metastases. Given that he was found to harbor an epidermal growth factor receptor (EGFR) mutation, he was also treated with the EGFRspecific tyrosine kinase inhibitor (TKI) gefitinib (250 mg/day). As a result of the development of adverse effects including fatigue, dermatitis, and liver dysfunction, however, the dose of gefitinib was initially reduced by half and treatment with the drug was subsequently discontinued after a total of 2 months. Computed tomography and magnetic resonance imaging revealed that the size of the original lesion and the number of brain metastases had increased. Treatment with the combination of cisplatin (125 mg) and pemetrexed (0.75 $\mathrm{mg})$ was initiated. After 8 weeks, this regimen was switched to vinorelbine (32 $\mathrm{mg}$ ) and docetaxel $(80 \mathrm{mg})$ because of the development of adverse effects. This latter treatment was also not tolerated well, and so administration of the EGFR-TKI erlotinib (150 mg/day) was initiated. The patient again experienced general adverse effects including dermal exanthema. At this time, he developed a foreign body sensation in both eyes and visited his local ophthalmologist, who referred him to the corneal service at Yamaguchi University Hospital for the treatment of corneal epithelial disorders with corneal thinning.

Slitlamp microscopy revealed bilateral superficial punctate keratopathy and conjunctival epithelial disorders. Corneal ulceration on his right eye was also observed, but no signs of infection or inflammation were apparent. His visual acuity was 20/25 OD and 20/25 OS. Schirmer's test detected bilateral mild hypolacrimation ( $5 \mathrm{~mm})$, and his corneal and conjunctival epithelial disorders were thus attributed to aqueous-deficient dry eye. We treated him by insertion of punctal plugs in both lower lacrimal puncta. Three weeks after plug insertion, his corneal and conjunctival epithelial disorders had apparently improved. However, 2 months after his first visit to our clinic, he was referred to us again for the treatment of bilateral corneal ulcers, which were confirmed by slitlamp microscopy (fig. 1). Slight stromal edema, ulceration with an opaque epithelium, and a shallow anterior chamber were also observed in the right eye. The next day, the anterior chamber of the right eye was flat, and so we treated this eye with a bandage soft contact lens. The right anterior chamber remained flat, however, and the patient was diagnosed with noninfectious corneal perforation of unknown cause and was prepared for tectonic keratoplasty. The oral administration of erlotinib was interrupted in preparation for general anesthesia, and the patient was treated with gatifloxacin eyedrops only. Two days later, the anterior chamber of his right eye had spontaneously reformed and the epithelial defects of both eyes had healed (fig. 2). The keratoplasty was therefore canceled and we monitored the patient carefully, but his corneal condition remained stable. Treatment with erlotinib at half the original dose was reinitiated and both eyes were maintained by the administration of artificial tears, with the punctal plugs remaining in place. Corneal ulceration or other corneal disorders did not recur.

\section{Discussion}

We present the case of corneal perforation that underwent spontaneous healing after a temporary suspension of the oral administration of erlotinib for the treatment of metastatic lung cancer. The clinical course of this case suggests that erlotinib might be secreted into tear fluid and thereby affect the condition of the corneal epithelium. 
Morishige et al.: Spontaneous Healing of Corneal Perforation after Temporary Discontinuation of Erlotinib Treatment

The clinical course of the present patient was characterized by the development of corneal epithelial disorders after the onset of erlotinib treatment, the temporary improvement of these disorders after the insertion of punctal plugs for treatment of dry eye, the subsequent development of corneal ulceration, the spontaneous healing of corneal perforation, and corneal epithelial disorders after cessation of erlotinib administration as well as the maintenance of a healthy cornea after the resumption of erlotinib at a reduced dose. This course suggested that erlotinib may have been secreted into the tear fluid of the patient and thereby adversely affected the corneal epithelium, and that punctal plug insertion may have increased the concentration of erlotinib in the tear fluid and eventually worsened its effects on the ocular surface. The treatment of dry eye by pooling of tear fluid may thus have exacerbated the clinical course in this case. Administration of artificial tears, which would remove secreted drugs from the ocular surface, may thus be preferable to punctal plug insertion for the amelioration of dry eye in patients treated with EGFR-TKIs. The dose of the latter drugs may need to be reduced in patients who develop corneal epithelial disorders.

The development of corneal perforation in the present patient was likely attributable to erlotinib exposure, but the etiology of the condition is unclear. Various proteinases released from bacteria [1], infiltrated neutrophils [2, 3], or resident keratocytes [4] degrade collagen in the corneal stroma of patients with infectious corneal ulcer. However, we did not observe any clinical signs suggestive of corneal infection or inflammation in the present patient. It is thus possible that erlotinib or other factors in tear fluid stimulated the release of proteinases from resident keratocytes and thereby gave rise to degradation of stromal collagen secondary to the epithelial defects.

Previous studies have described corneal disorders in patients treated with erlotinib, including a case of corneal perforation that required treatment with tectonic keratoplasty [5], a case of corneal melting that was successfully treated with autologous serum eyedrops [6], and a case of persistent epithelial defects [7]. Laboratory investigations [8,9] have also suggested that inhibition of EGFR function by drugs such as erlotinib may adversely affect the corneal epithelium. The recent introduction of EGFR-TKIs for the treatment of various types of cancer might be expected to result in an increase in the number of patients with corneal disorders induced by these drugs.

TS-1, a combination of tegafur, gimeracil, and oteracil potassium, is widely administered for the treatment of various types of cancer in Japan. The widespread application of this drug has resulted in the development of lacrimal duct dysfunction [10] or corneal epithelial disorders [11] in many patients. These various observations and the present case therefore suggest that individuals treated with certain anticancer drugs should be monitored for the development of ocular surface disorders.

\section{References}

1 Kreger AS, Gray LD: Purification of Pseudomonas aeruginosa proteases and microscopic characterization of pseudomonal protease-induced rabbit corneal damage. Infect Immun 1978;19:630-648.

-2 Okada Y, Nakanishi I: Activation of matrix metalloproteinase 3 (stromelysin) and matrix metalloproteinase 2 ('gelatinase') by human neutrophil elastase and cathepsin G. FEBS Lett 1989;249:353-356.

-3 Steuhl KP, Doring G, Henni A, Thiel HJ, Botzenhart K: Relevance of host-derived and bacterial factors in Pseudomonas aeruginosa corneal infections. Invest Ophthalmol Vis Sci 1987;28:1559-1568.

4 Nagano T, Hao JL, Nakamura M, et al: Stimulatory effect of pseudomonal elastase on collagen degradation by cultured keratocytes. Invest Ophthalmol Vis Sci 2001;42:1247-1253.

5 Saint-Jean A, Sainz de la Maza M, Morral M, et al: Ocular adverse events of systemic inhibitors of the epidermal growth factor receptor: report of 5 cases. Ophthalmology 2012;119:1798-1802.

6 Chow VW, Jhanji V, Chi SC: Erlotinib-related corneal melting. Ophthalmology 2013;120:1104.e1. 
Case Reports in

Ophthalmology

\begin{tabular}{l|l}
\hline Case Rep Ophthalmol 2014;5:6-10 \\
\hline DOI: 10.1159/000358082 & $\begin{array}{l}\text { @ 2014 S. Karger AG, Basel } \\
\text { www.karger.com/cop }\end{array}$ \\
\hline
\end{tabular}

Morishige et al.: Spontaneous Healing of Corneal Perforation after Temporary Discontinuation of Erlotinib Treatment

7 Johnson KS, Levin F, Chu DS: Persistent corneal epithelial defect associated with erlotinib treatment. Cornea 2009;28:706-707.

8 Nakamura Y, Sotozono C, Kinoshita S: The epidermal growth factor receptor (EGFR): role in corneal wound healing and homeostasis. Exp Eye Res 2001;72:511-517.

-9 Wilson SE, He YG, Weng J, Zieske JD, Jester JV, Schultz GS: Effect of epidermal growth factor, hepatocyte growth factor, and keratinocyte growth factor, on proliferation, motility and differentiation of human corneal epithelial cells. Exp Eye Res 1994;59:665-678.

$\checkmark 10$ Esmaeli B, Golio D, Lubecki L, Ajani J: Canalicular and nasolacrimal duct blockage: an ocular side effect associated with the antineoplastic drug S-1. Am J Ophthalmol 2005;140:325-327.

$\checkmark 11$ Kobashi H, Kamiya K, Shimizu K: A case of corneal epithelial lesion and keratoconjunctival pigmentation due to anticancer drug S-1. Jpn J Ophthalmol 2011;55:163-165.

N.M. and N.H. contributed equally to this report.
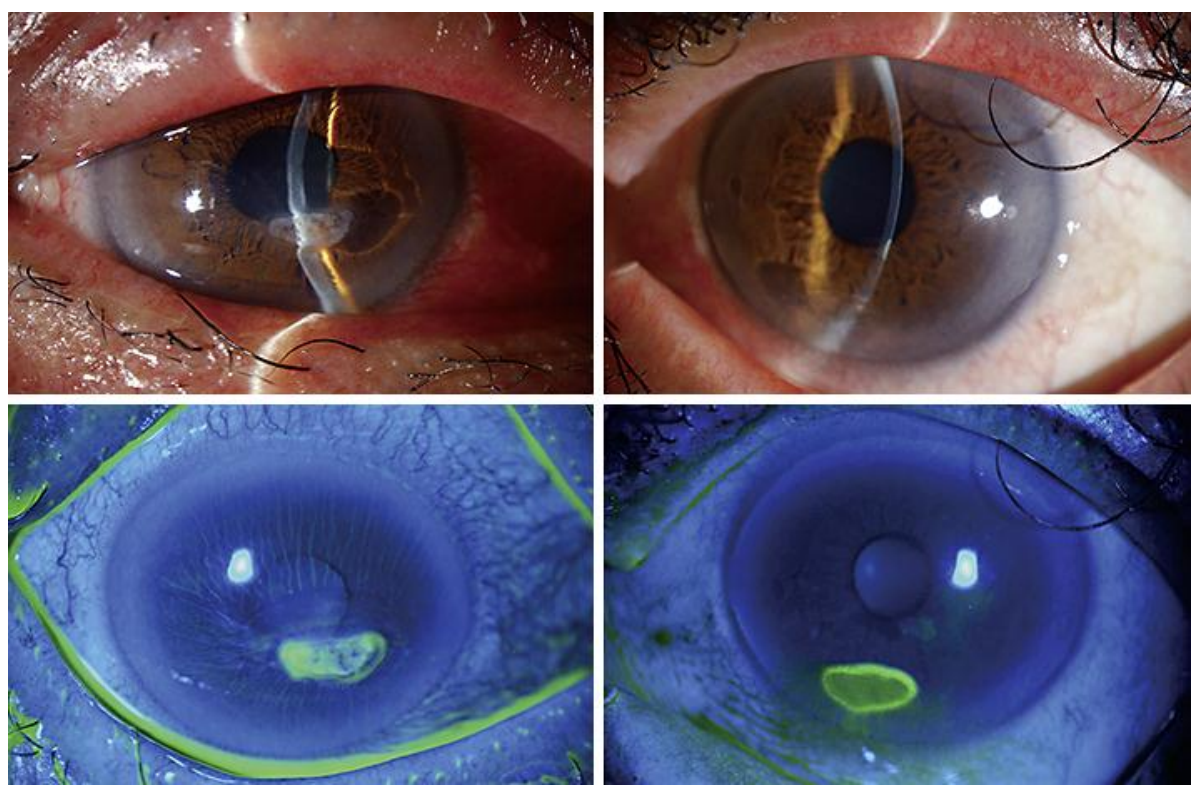

Fig. 1. Slitlamp photographs of the corneas of the patient on his second visit to our clinic. The anterior chamber of the right eye was shallower than that of the left eye. Fluorescein staining revealed bilateral corneal ulceration. 
Case Reports in

Ophthalmology

\begin{tabular}{l|l}
\hline Case Rep Ophthalmol 2014;5:6-10 \\
\hline DOI: 10.1159/000358082 & $\begin{array}{l}\text { ○ 2014 S. Karger AG, Basel } \\
\text { www.karger.com/cop }\end{array}$ \\
\hline
\end{tabular}

Morishige et al:: Spontaneous Healing of Corneal Perforation after Temporary Discontinuation of Erlotinib Treatment
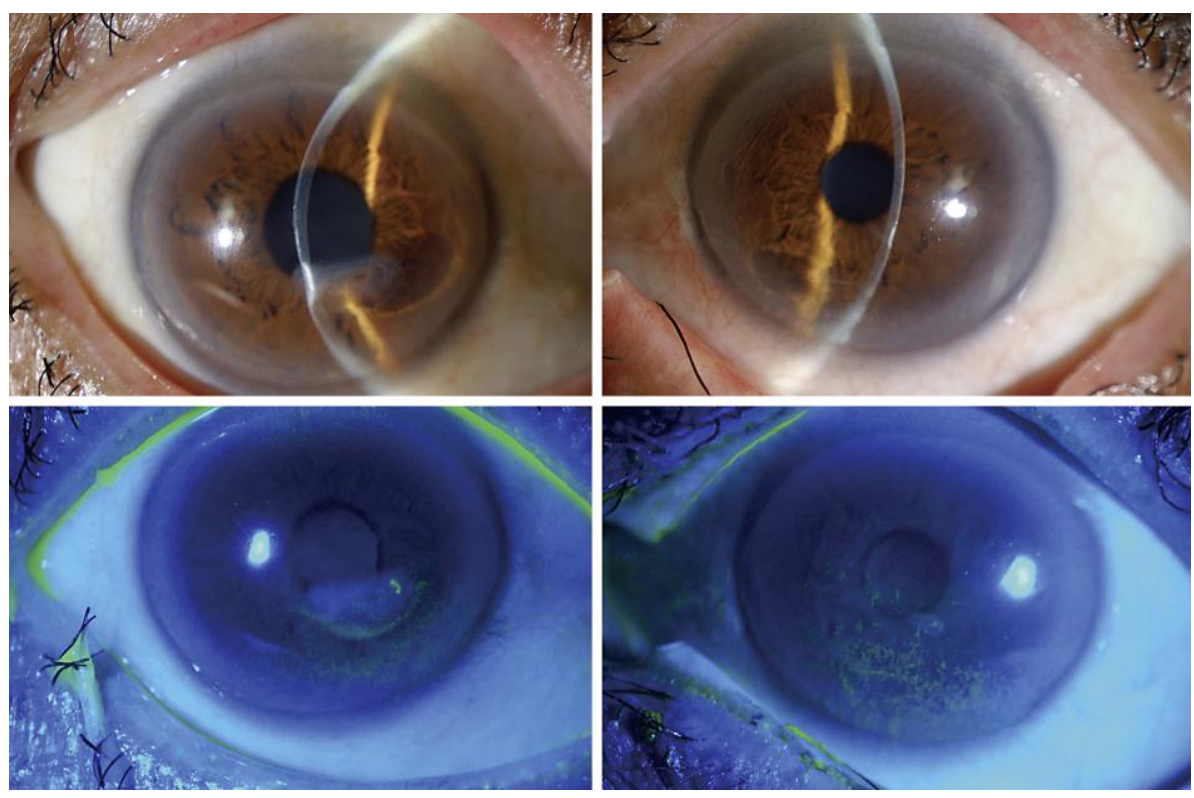

Fig. 2. Slitlamp photographs of the corneas of the patient obtained 2 days after cessation of erlotinib treatment. The anterior chamber of the right eye had reformed, and the bilateral corneal ulceration had improved. 